\title{
Generalized involute and evolute curves of equiform spacelike curves with a timelike equiform principal normal in $E_{1}^{3}$
}

\author{
A. Elsharkawy
}

\section{Correspondence:}

ayman_ramadan@science.tanta.edu.eg Mathematics Department, Faculty of Science Tanta University, Tanta, Egypt

\begin{abstract}
Equiform geometry is considered as a generalization of the other geometries. In this paper, involute and evolute curves are studied in the case of the curve $\alpha$ is an equiform spacelike with a timelike equiform principal normal vector $N$. Furthermore, the equiform frames of the involute and evolute curves are obtained. Also, the equiform curvatures of the involute and evolute curves are obtained in Minkowski 3-space.
\end{abstract}

Keywords: Minkowski 3-space, Involute, Evolute, Equiform geometry, Equiform curvatures

AMS Subject Classification: 53A35, 53C50

\section{Introduction}

In the last two decades, curves in Minkowski space have been studied by many mathematicians such as [1-3]. Specially, involute and evolute curves got an interest from alot of mathematicians in Minkowski 3-space. According to the usual Frenet frame, involute and evolute curves in Minkowski 3-space $E_{1}^{3}$ were defined and studied in $[1,2,4,5]$. The equiform geometry was defined in different spaces such as Galilean space [6], pseudoGalilean space [7], Euclidean space [8], isotropic space [9], and Minkowski space [3, 10, 11].

In this paper, we firstly introduce the equiform parameter, the equiform frame, and the equiform formulas in the case of equiform spacelike curves with a timelike equiform principal normal in Minkowski space $E_{1}^{3}$. Secondly, we introduce the involute and the evolute of the equiform spacelike curve with a timelike equiform principal normal. Further, the equiform frames for the involute and the evolute curves are obtained. Also, the equiform curvatures of the involute and the evolute curves are obtained.

\section{Preliminaries}

The three-dimensional Lorentzian space, or Minkowski 3 -space $E_{1}^{3}$, is the space $R^{3}$ equipped with the metric $g$ defined as:

$$
g(U, V)=-u_{1} v_{1}+u_{2} v_{2}+u_{3} v_{3}
$$

(c) The Author(s). 2020 Open Access This article is licensed under a Creative Commons Attribution 4.0 International License, which permits use, sharing, adaptation, distribution and reproduction in any medium or format, as long as you give appropriate credit to the original author(s) and the source, provide a link to the Creative Commons licence, and indicate if changes were made. The images or other third party material in this article are included in the article's Creative Commons licence, unless indicated otherwise in a credit line to the material. If material is not included in the article's Creative Commons licence and your intended use is not permitted by statutory regulation or exceeds the permitted use, you will need to obtain permission directly from the copyright holder. To view a copy of this licence, visit http://creativecommons.org/licenses/by/4.0/. 
where $U=\left(u_{1}, u_{2}, u_{3}\right)$ and $V=\left(v_{1}, v_{2}, v_{3}\right)$ are any two vectors in $R^{3}$. The vector $U$ in $E_{1}^{3}$ may be lightlike if $g(U, U)=0$ and $U \neq 0$ or spacelike if $g(U, U)>0$ or $U=0$ or timelike if $g(U, U)<0$. The norm (length) of the vector $U$ is defined by $\|U\|=\sqrt{|g(U, U)|}$.

The Lorentzian cross product is given by:

$U \wedge V=\left(u_{3} v_{2}-u_{2} v_{3}, u_{3} v_{1}-u_{1} v_{3}, u_{1} v_{2}-u_{2} v_{1}\right)$,

where $U$ and $V \in E_{1}^{3}[12,13]$.

A differentiable map $\alpha: I \subset R \rightarrow E_{1}^{3}$ is called smooth curve in Minkowski 3-space, where $I$ is an open interval. Suppose that $\{t(s), n(s), b(s)\}$ be the orthonormal Frenet frame along the curve $\alpha(s)$, where $t(s), n(s)$, and $b(s)$ are the tangent, the principal normal, and the binormal vectors of the curve $\alpha$, respectively.

Any curve $\alpha$ in Minkowski 3-space can be one of the following cases and below the corresponding Frenet formulas:

(1) $\alpha$ is a spacelike curve with

(i) a spacelike principal normal, then Frenet formulas are given by:

$$
\begin{aligned}
& \frac{d t}{d s}=\dot{t}=k n, \frac{d n}{d s}=\dot{n}=-k t+\tau b, \frac{d b}{d s}=\dot{b}=\tau n . \\
& g(t, t)=g(n, n)=1, g(b, b)=-1, \\
& g(t, n)=g(n, b)=g(b, t)=0 .
\end{aligned}
$$

(ii) a timelike principal normal, then Frenet formulas are given by:

$$
\begin{aligned}
& \dot{t}=k n, \dot{n}=k t+\tau b, \dot{b}=\tau n, \\
& g(t, t)=g(b, b)=1, g(n, n)=-1, \\
& g(t, n)=g(n, b)=g(b, t)=0 .
\end{aligned}
$$

(iii) a null (lightlike) principal normal, then Frenet formulas are given by:

$$
\begin{aligned}
& \dot{t}=k n, \dot{n}=\tau n, \dot{b}=-k t-\tau b, \\
& g(t, t)=1, g(n, n)=g(b, b)=0, g(n, b)=1, \\
& g(t, n)=g(t, b)=0 .
\end{aligned}
$$

(2) $\alpha$ is a timelike curve, then Frenet formulas are given by:

$$
\begin{aligned}
& \dot{t}=k n, \dot{n}=k t+\tau b, \dot{b}=-\tau n, \\
& g(t, t)=-1, g(n, n)=g(b, b)=1, g(n, b)=1, \\
& g(t, n)=g(t, b)=0 .
\end{aligned}
$$

(3) $\alpha$ is a lightlike curve, then Frenet formulas are given by: 


$$
\begin{aligned}
& \dot{t}=k n, \dot{n}=\tau t-k b, \dot{b}=-\tau n, \\
& g(n, n)=1, g(t, t)=g(b, b)=0, g(t, b)=1, \\
& g(t, n)=g(n, b)=0 .
\end{aligned}
$$

[13]

The equiform geometry has minor importance related to usual one, and the curves that appear here in equiform geometry can be seen as a generalization of well-known curves from other geometries.

Let $\gamma(s)=t(s)$ be the spherical tangent indicatrix of the curve $\alpha$ and $\sigma$ be an arc length parameter of $\gamma$. We can make a reparameterization of $\alpha$ by the parameter $\sigma, \alpha=\alpha(\sigma)$ : $I \rightarrow E_{1}^{3}$, the parameter $\sigma$ is called the equiform parameter, of the curve $\alpha(\sigma)$.

Let $\sigma$ be the arc length parameter of spherical tangent indicatrix $\zeta$, then we have:

$$
\begin{aligned}
& \left\|\frac{d \zeta}{d \sigma} \frac{d \sigma}{d s}\right\|=\|\kappa n\|, \\
& \frac{d \sigma}{d s}=\kappa=\frac{1}{\rho} .
\end{aligned}
$$

By integration with respect to $s$, we have:

$$
\sigma=\int \frac{d s}{\rho}
$$

where $\rho$ is the radius of curvature of $\alpha$ [11].

Let $T, N$, and $B$ be the orthogonal equiform frame along the curve $\alpha(\sigma)$ in Minkowski 3-space, where $T, N$, and $B$ are the equiform-tangent, the equiform-normal, and the equiform-binomial vectors of the curve $\alpha(\sigma)$, respectively. They are given by $T=\frac{d \alpha}{d \sigma}=$ $\rho t, N=\rho n, b=\rho b[10,11]$.

The function $K_{1}: I \rightarrow R$ defined by $K_{1}=\frac{d \rho}{d s}$ is called the first equiform curvature of $\alpha(\sigma)$, and the function $K_{2}: I \rightarrow R$ defined by $K_{2}=\frac{\tau}{\kappa}$ is the called second equiform curvature of $\alpha(\sigma)$.

Definition 1 A curve $\alpha(\sigma)$ is an equiform spacelike if $g(T, T)=\rho^{2}>0$ or $T=0$, equiform timelike if $g(T, T)=-\rho^{2}<0$, or equiform null if $g(T, T)=0$ and $T \neq 0$.

If $\alpha(\sigma)$ is an equiform spacelike with a timelike equiform principal normal vector, then the equiform formulas are given in [10] by:

$$
\begin{aligned}
& \frac{d T}{d \sigma}=T^{\prime}=K_{1} T+N, \\
& \frac{d N}{d \sigma}=N^{\prime}=T+K_{1} N+K_{2} B, \\
& \frac{d B}{d \sigma}=B^{\prime}=K_{2} N+K_{1} B,
\end{aligned}
$$

where

$$
\begin{aligned}
& g(T, T)=-g(N, N)=g(B, B)=\rho^{2}, \\
& g(T, N)=g(N, B)=g(B, T)=0 .
\end{aligned}
$$

Lemma 1 Suppose that a curve $\alpha$ is an equiform spacelike with a timelike equiform principal normal $N$. If $\alpha(\sigma)$ is parameterized by the equiform parameter $\sigma$, then:

$$
T=\frac{d \alpha}{d \sigma}, N=\frac{B \wedge T}{\rho}, B=-\frac{\alpha^{\prime} \wedge \alpha^{\prime \prime}}{\rho} .
$$


Lemma 2 If a curve $\alpha\left(\sigma^{*}\right)$ is an equiform spacelike with a timelike equiform principal normal $N$ and $\sigma^{*}$ is not necessary the equiform parameter of the curve $\alpha$, then:

$$
T=\frac{\rho \frac{d \alpha}{d \sigma^{*}}}{\left\|\frac{d \alpha}{d \sigma^{*}}\right\|}, N=\frac{B \wedge T}{\rho}, B=-\frac{\rho\left(\frac{d \alpha}{d \sigma^{*}} \wedge \frac{d^{2} \alpha}{d \sigma^{* 2}}\right)}{\left\|\frac{d \alpha}{d \sigma^{*}} \wedge \frac{d^{2} \alpha}{d \sigma^{* 2}}\right\|}
$$

Lemma 3 Suppose that a curve $\alpha$ is an equiform spacelike with a timelike equiform principal normal $N$. Then, the equiform curvatures are given by:

$$
\begin{aligned}
& K_{1}=\frac{g\left(T^{\prime}, T\right)}{\rho^{2}}=\frac{-g\left(N^{\prime}, N\right)}{\rho^{2}}=\frac{g\left(B^{\prime}, B\right)}{\rho^{2}}, \\
& K_{2}=\frac{g\left(N^{\prime}, B\right)}{\rho^{2}}=\frac{-g\left(B^{\prime}, N\right)}{\rho^{2}} .
\end{aligned}
$$

Definition 2 A curve $\alpha(\sigma)$ is an ordinary helix if the second equiform curvature $K_{2}=0$, and it is a general helix if $K_{2}$ is constant.

\section{The involute of an equiform spacelike curve with a timelike equiform principal normal}

In this section, we study the involute curve of the equiform spacelike curve with a timelike equiform principal normal vector $N$ in $E_{1}^{3}$. Also, the equiform frame of the involute curve is introduced. Furthermore, the equiform curvatures of the involute curve are obtained.

Definition 3 Let $\alpha(\sigma)$ be an equiform spacelike curve with a timelike equiform principal normal and a curve $\beta(\sigma)$ be given, then the curve $\beta$ is called an involute of the curve $\alpha$, if the tangent at the point $\alpha(\sigma)$ to the curve $\alpha$ passes through the tangent at the point $\beta(\sigma)$ to the curve $\beta$. In the other words, $\beta(\sigma)$ is an involute of $\alpha(\sigma)$ if the equation $g\left(T, T^{*}\right)=0$ is satisfied. $\beta(\sigma)$ can be written in terms of the curve $\alpha$ as:

$$
\beta(\sigma)=\alpha(\sigma)+\lambda(\sigma) T(\sigma)
$$

Let the equiform frames of the curve $\alpha(\sigma)$ and $\beta(\sigma)$ be $\{T, N, B\}$ and $\left\{T^{*}, N^{*}, B^{*}\right\}$, respectively.

Theorem 1 Let $\alpha(\sigma)$ be an equiform spacelike curve with a timelike equiform principal normal and suppose that a curve $\beta(\sigma)$ is the involute of the curve $\alpha$. Then,

$$
\beta(\sigma)=\alpha(\sigma)+\frac{c-s}{\rho} T(\sigma),
$$

where $c$ is constant.

Proof Suppose that $\beta(\sigma)$ is the involute of $\alpha(\sigma)$. Then, we can write $\beta(\sigma)$ as:

$$
\beta(\sigma)=\alpha(\sigma)+\lambda(\sigma) T(\sigma) .
$$

By taking the derivative of Eq. (1), with respect to $\sigma$, we have:

$$
\frac{d \beta}{d \sigma}=\left(1+\lambda(\sigma) K_{1}+\lambda^{\prime}(\sigma)\right) T+\lambda(\sigma) N .
$$

Since $g\left(T^{*}, T\right)=0$, then we have the differential equation:

$$
\lambda^{\prime}(\sigma)+\lambda(\sigma) K_{1}+1=0
$$


Hence,

$$
\lambda=\frac{c-s}{\rho} .
$$

From Eqs. (1) and (2), we obtain:

$$
\beta(\sigma)=\alpha(\sigma)+\frac{1}{\rho}(c-s) T(\sigma) .
$$

Corollary 1 The distance between the curve $\alpha(\sigma)$ and its involute $\beta(\sigma)$ is $|c-s|$.

Theorem 2 Let $\alpha(\sigma)$ be an equiform spacelike curve with a timelike equiform principal normal and suppose that a curve $\beta$ is an involute of the curve $\alpha$, then:

$$
\left[\begin{array}{c}
T^{*} \\
N^{*} \\
B^{*}
\end{array}\right]=\frac{\rho^{*}}{\rho}\left[\begin{array}{ccc}
0 & 1 & 0 \\
\frac{-1}{\sqrt{K_{2}^{2}+1}} & 0 & \frac{-K_{2}}{\sqrt{K_{2}^{2}+1}} \\
\frac{-K_{2}}{\sqrt{K_{2}^{2}+1}} & 0 & \frac{1}{\sqrt{K_{2}^{2}+1}}
\end{array}\right]\left[\begin{array}{c}
T \\
N \\
B
\end{array}\right] .
$$

Proof By taking the derivative of Eq. (3) with respect to $\sigma$, we have:

$$
\frac{d \beta}{d \sigma}=\frac{c-s}{\rho} N, \quad \quad\left\|\frac{d \beta}{d \sigma}\right\|=|c-s| .
$$

Then,

$$
T^{*}(\sigma)=\frac{\rho^{*} \frac{d \beta}{d \sigma}}{\left\|\frac{d \beta}{d \sigma}\right\|}= \pm \frac{\rho^{*}}{\rho} N .
$$

Let us assume that:

$$
T^{*}=\frac{\rho^{*}}{\rho} N \text {. }
$$

By taking the derivative of Eq. (4) with respect to $\sigma$, we have:

$$
\frac{d^{2} \beta}{d \sigma^{2}}=\frac{(c-s)}{\rho} T-N+\frac{(c-s) K_{2}}{\rho} B .
$$

Hence, we have:

$$
\begin{aligned}
& \frac{d \beta}{d \sigma} \wedge \frac{d^{2} \beta}{d \sigma^{2}}=\frac{(c-s)^{2}}{\rho}\left[-K_{2} T+B\right], \\
& \left\|\frac{d \beta}{d \sigma} \wedge \frac{d^{2} \beta}{d \sigma^{2}}\right\|=(c-s)^{2} \sqrt{K_{2}^{2}+1} . \\
& B^{*}=\frac{\rho^{*}\left(\frac{d \beta}{d \sigma} \wedge \frac{d^{2} \beta}{d \sigma^{2}}\right)}{\left\|\frac{d \beta}{d \sigma} \wedge \frac{d^{2} \beta}{d \sigma^{2}}\right\|}=\frac{\rho^{*}}{\rho \sqrt{K_{2}^{2}+1}}\left[-K_{2} T+B\right] .
\end{aligned}
$$

Since $N^{*}=-\frac{B^{*} \wedge T^{*}}{\rho^{*}}$, then we obtain:

$$
N^{*}=\frac{\rho^{*}}{\rho \sqrt{K_{2}^{2}+1}}\left[-T-K_{2} B\right] .
$$

Corollary 2 If $\alpha(\sigma)$ is an equiform spacelike curve with a timelike equiform principal normal vector, then its involutes are equiform timelike curves. 
Theorem 3 Let $\beta(\sigma)$ be an involute of the curve $\alpha(\sigma)$, and $K_{1}^{*}, K_{2}^{*}$ be the first and second equiform curvatures of the curve $\beta$, respectively. Then, $K_{1}^{*}$ and $K_{2}^{*}$ are given respectively by:

$$
K_{1}^{*}=\frac{-\rho}{|c-s|} \frac{d \rho^{*}}{d s}, \quad K_{2}^{*}=\frac{-\rho^{*} K_{2}^{\prime}}{|c-s|\left(K_{2}^{2}+1\right)} .
$$

Proof Since $T^{*}=\frac{d \beta}{d \sigma^{*}}=\frac{d \beta}{d \sigma} \frac{d \sigma}{\sigma^{*}}$. Using Eq. (4), we obtain:

$$
\frac{d \sigma}{d \sigma^{*}}=\frac{\rho^{*}}{|c-s|} \text {. }
$$

By taking the derivative Eq. (5) and using Eq. (11), we get:

$$
\begin{aligned}
& \frac{d T^{*}}{d \sigma^{*}}=T^{* \prime}=\left(\frac{\rho^{*}}{\rho} T+\frac{d \rho^{*}}{d s} N+\frac{K_{2} \rho^{*}}{\rho} B\right)\left(\frac{\rho^{*}}{|c-s|}\right), \\
& g\left(T^{* \prime}, T^{*}\right)=\frac{\rho^{* 2} \rho}{|c-s|} \frac{d \rho^{*}}{d s} .
\end{aligned}
$$

Therefore, the first equiform curvature $K_{1}^{*}=\frac{-g\left(T^{*}, T^{*}\right)}{\rho^{* 2}}$ is given by:

$$
K_{1}^{*}=\frac{-\rho}{|c-s|} \frac{d \rho^{*}}{d s} .
$$

Now, suppose that Eq. (10) is:

$$
N^{*}=-a T-a K_{2} B,
$$

where $a=\frac{\rho^{*}}{\rho \sqrt{K_{2}^{2}+1}}$. Taking the derivative of the above equation with respect to $\sigma^{*}$, we have:

$$
\begin{aligned}
& N^{* \prime}=\left[-\left(a K_{1}+\frac{d a}{d \sigma}\right) T+\left(-a-a K_{2}^{2}\right) N\right. \\
& \left.-\left(a K_{1} K_{2}+a \frac{d K_{2}}{d \sigma}+\frac{d a}{d \sigma} K_{2}\right) B\right] \frac{\rho^{*}}{|c-s|}, \\
& g\left(N^{* \prime}, B^{*}\right)=\frac{-\rho^{2} \rho^{*}}{|c-s|} a^{2} K_{2}^{\prime} .
\end{aligned}
$$

Thus, the second equiform curvature $K_{2}^{*}=\frac{g\left(N^{* \prime}, B^{*}\right)}{\rho^{* 2}}$ is given by:

$$
K_{2}^{*}=\frac{-\rho^{*} K_{2}^{\prime}}{|c-s|\left(K_{2}^{2}+1\right)} .
$$

Corollary 3 If $\alpha(\sigma)$ is an equiform spacelike curve with a timelike equiform principal normal $N$ and $\beta(\sigma)$ is an involute of $\alpha(\sigma)$, then:

1. If $\alpha(\sigma)$ is a planar curve, then $\beta(\sigma)$ is also planar.

2. If $\alpha(\sigma)$ is an ordinary helix $\left(K_{2}=0\right)$, then $\beta(\sigma)$ is planar.

3. If $\alpha(\sigma)$ is a general helix $\left(K_{2}=c\right)$, then $\beta(\sigma)$ is planar.

Proof The proofs come forward from the equation of $K_{2}^{*}$. 


\section{The evolute of equiform spacelike curve with a timelike equiform principal normal}

In this section, the evolute curves of the equiform spacelike curve with a timelike equiform principal normal $N$ are studied in $E_{1}^{3}$. Moreover, the equiform frame of the evolute curve is introduced. Furthermore, the equiform curvatures of the evolute are computed.

Definition 4 Let $\alpha(\sigma)$ be an equiform spacelike with a timelike equiform principal normal and a curve $\gamma$ with the same interval be given. For $\forall \sigma \in I$, if the tangent at the point $\gamma(\sigma)$ to the curve $\gamma(\sigma)$ passes through the point $\alpha(s)$ and

$$
g\left(T^{*}(\sigma), T(\sigma)\right)=0,
$$

then $\gamma(\sigma)$ is called an evolute of the curve $\alpha(\sigma)$.

Let the Frenet frame of the curve $\alpha(\sigma)$ and $\gamma(\sigma)$ be $\{T, N, B\}$ and $\left\{T^{*}, N^{*}, B^{*}\right\}$, respectively.

Theorem 4 Let $\alpha(\sigma)$ be an equiform spacelike with a timelike equiform principal normal and a curve $\gamma(\sigma)$ be an evolute of $\alpha$, then:

$$
\gamma(\sigma)=\alpha(\sigma)-N(\sigma)+\tanh \left(\int K_{2} d \sigma+c\right) B(\sigma)
$$

where $c \in R$

and

$$
\frac{d \sigma}{d \sigma^{*}}=\frac{\rho^{*} \cosh \left(\int K_{2} d \sigma+c\right)}{\rho\left|-K_{1}+K_{2} \tanh \left(\int K_{2} d \sigma+c\right)\right|} .
$$

Proof Suppose that a curve $\gamma(\sigma)$ be the evolute of the curve $\alpha(\sigma)$. Then, the vector $\gamma(\sigma)-\alpha(\sigma)$ is perpendicular to the vector $T(\sigma)$. Then,

$$
\gamma(\sigma)-\alpha(\sigma)=\lambda(\sigma) N(\sigma)+\mu B(\sigma) .
$$

By taking the derivative of Eq. (12) with respect to $\sigma$, we have:

$$
\begin{aligned}
& \frac{d \gamma}{d \sigma}=[1+\lambda(\sigma)] T+\left[\lambda(\sigma) K_{1}+\lambda^{\prime}(\sigma)+\mu K_{2}\right] N \\
& +\left[\lambda(\sigma) K_{2}+\mu K_{1}+\mu^{\prime}\right] B .
\end{aligned}
$$

Then, we get:

$$
\begin{aligned}
& g\left(\frac{d \gamma}{d \sigma}, T\right)=[1+\lambda(\sigma)] g(T, T) \\
& {\left[\left(\lambda(\sigma) K_{1}+\lambda^{\prime}(\sigma)+\mu K_{2}\right] g(N, T)\right.} \\
& +\left[\lambda(\sigma) K_{2}+\mu K_{1}+\mu^{\prime}\right] g(B, T) .
\end{aligned}
$$


Since $g\left(\frac{d \gamma}{d \sigma}, T\right)=0$, then we have:

$$
\lambda(\sigma)=-1,
$$

and hence,

$$
\frac{d \gamma}{d \sigma}=\left(-K_{1}+\mu K_{2}\right) N+\left(\mu^{\prime}+\mu K_{1}-K_{2}\right) B
$$

From Eqs. (12) and (14), the vector $\frac{d \gamma}{d \sigma}$ is parallel to the vector $\gamma-\alpha$, and we have:

$$
\frac{-K_{1}+\mu K_{2}}{\lambda(\sigma)}=\frac{\mu^{\prime}+\mu K_{1}-K_{2}}{\mu} .
$$

Also, we have:

$$
K_{2}=\frac{\mu^{\prime}}{1-\mu^{2}} .
$$

By taking the integration of the last equation, we get:

$$
\int K_{2} d \sigma+c=\tanh ^{-1}(\mu(\sigma)) .
$$

Hence, we find:

$$
\mu(\sigma)=\tanh \left(\int K_{2} d \sigma+c\right) .
$$

By substituting from Eqs. (13) and (15) into Eq. (12), we have:

$$
\gamma(\sigma)=\alpha(\sigma)-N(\sigma)+\tanh \left(\int K_{2} d \sigma+c\right) B(\sigma) .
$$

Since,

$$
\begin{aligned}
& T^{*}=\gamma^{\prime}=\left[-K_{1}+K_{2} \tanh \left(\int K_{2} d \sigma+c\right)\right] . \\
& \cdot\left[N-\tanh \left(\int K_{2} d \sigma+c\right) B\right] \frac{d \sigma}{d \sigma^{*}}
\end{aligned}
$$

and

$$
\begin{aligned}
& g\left(T^{*}, T^{*}\right)=\left[-K_{1}+K_{2} \tanh \left(\int K_{2} d \sigma+c\right)\right]^{2} \\
& . \rho^{2} \operatorname{sech}^{2}\left(\int K_{2} d \sigma+c\right)\left(\frac{d \sigma}{d \sigma^{*}}\right)^{2}
\end{aligned}
$$

moreover, we get:

$$
\frac{d \sigma}{d \sigma^{*}}=\frac{\rho^{*} \cosh \left(\int K_{2} d \sigma+c\right)}{\rho\left|-K_{1}+K_{2} \tanh \left(\int K_{2} d \sigma+c\right)\right|} .
$$

Theorem 5 Let $\gamma: I \rightarrow E_{1}^{3}$ be the evolute curve of the equiform spacelike curve $\alpha: I \rightarrow$ $E_{1}^{3}$. Then, the equiform frame of the curve $\gamma$ is given by:

$$
\left[\begin{array}{c}
T^{*} \\
N^{*} \\
B^{*}
\end{array}\right]=\frac{\rho^{*}}{\rho}\left[\begin{array}{ccc}
0 & \cosh z & -\sinh z \\
-1 & 0 & 0 \\
0 & -\sinh z & \cosh z
\end{array}\right]\left[\begin{array}{c}
T \\
N \\
B
\end{array}\right],
$$


where $z=\left(\int K_{2} d \sigma+c\right)$.

Proof By similar proof of Theorem 2, we obtain the required.

Corollary 4 If the curve $\alpha$ is a equiform spacelike curve with a timelike equiform principal normal, then its evolutes are equiform timelike curves.

Proof The proof comes forward from Theorem 5.

Theorem 6 Let the curve $\gamma$ be an evolute of the curve $\alpha$ and let $K_{1}^{*}$ and $K_{2}^{*}$ be the first and second equiform curvetures of the curve $\gamma$. Then,

$$
\begin{aligned}
& K_{1}^{*}=\frac{d \rho^{*}}{d s} \frac{\left|\cosh \left(\int K_{2} d \sigma+c\right)\right|}{\left|-K_{1}+K_{2} \tanh \left(\int K_{2} d \sigma+c\right)\right|}, \\
& K_{2}^{*}=\frac{\rho^{*}\left|\sinh 2\left(\int K_{2} d \sigma+c\right)\right|}{2 \rho\left|-K_{1}+K_{2} \tanh \left(\int K_{2} d \sigma+c\right)\right|} .
\end{aligned}
$$

Proof By taking the derivative of $N^{*}$ with respect to $\sigma^{*}$, we have:

$$
\begin{aligned}
& N^{* \prime}=\left[\frac{\rho^{*}\left|\cosh \left(\int K_{2} d \sigma+c\right)\right|}{\rho\left|-K_{1}+K_{2} \tanh \left(\int K_{2} d \sigma+c\right)\right|}\right] \\
& \cdot\left[\frac{-d \rho^{*}}{d s} T-\frac{\rho^{*}}{\rho} N\right] .
\end{aligned}
$$

Then,

$$
g\left(N^{* \prime}, N^{*}\right)=\frac{d \rho^{*}}{d s} \frac{\rho^{* 2}\left|\cosh \left(\int K_{2} d \sigma+c\right)\right|}{\left|-K_{1}+K_{2} \tanh \left(\int K_{2} d \sigma+c\right)\right|} .
$$

Therefore,

$$
K_{1}^{*}=\frac{d \rho^{*}}{d s} \frac{\left|\cosh \left(\int K_{2} d \sigma+c\right)\right|}{\left|-K_{1}+K_{2} \tanh \left(\int K_{2} d \sigma+c\right)\right|} .
$$

Also,

$$
g\left(N^{* \prime}, B^{*}\right)=\frac{-\rho^{* 3}\left|\cosh \left(\int K_{2} d \sigma+c\right)\right| \sinh \left(\int K_{2} d \sigma+c\right)}{\rho\left|K_{1}-K_{2} \tanh \left(\int K_{2} d \sigma+c\right)\right|} .
$$

Thus,

$$
\begin{aligned}
& K_{2}^{*}=\frac{g\left(N^{* \prime}, B^{*}\right)}{\rho^{* 2}} \\
& =\frac{-\rho^{*} \cosh \left(\int K_{2} d \sigma+c\right) \mid \sinh \left(\int K_{2} d \sigma+c\right)}{\rho\left|-K_{1}+K_{2} \tanh \left(\int K_{2} d \sigma+c\right)\right|} .
\end{aligned}
$$

Therefore,

$$
K_{2}^{*}=\frac{-\rho^{*}\left|\sinh 2\left(\int K_{2} d \sigma+c\right)\right|}{2 \rho\left|-K_{1}+K_{2} \tanh \left(\int K_{2} d \sigma+c\right)\right|} .
$$

Corollary 5 If the curve $\alpha(\sigma)$ is planar, then its evolute curve $\beta(\sigma)$ is also planar. 
Acknowledgements

Not applicable.

\section{Authors' contributions}

The author collected the data, performed the calculations, and was a major contributor in writing the manuscript.

Funding

Not applicable.

\section{Availability of data and materials}

The datasets used and/or analyzed during the current study are available from the corresponding author on reasonable request.

\section{Competing interests}

The author declares that he has no competing interests.

Received: 5 August 2019 Accepted: 28 April 2020

Published online: 29 May 2020

\section{References}

1. Bahaddin, B., Karacan, M.: On the involute and evolute curves of the timelike curve in Minkowski 3-space. Demonstratio Math. (2007). https://doi.org/10.1515/dema-2007-0320

2. Bahaddin, B., Karacan, M.: On involute and evolute curves of spacelike curve with a spacelike principal normal in Minkowski 3-space. Int. J. Math. Combin. 1, 27-37 (2009)

3. Solouma, E.: Special equiform Smarandache curves in Minkowski space-time. J. Egy. Math. Soc. 25(3), 319-325 (2017)

4. Bilici, M.: On the involutes of the spacelike curve with a timelike binormal in Minkowski 3-space. Int. Math. Forum. 4(31), 1497-1509 (2009)

5. Ozturk, U., Ozturk, E., llarslan, K.: On the involute-evolute of the pseudonull curve in Minkowski 3-space. J. Appl. Math., 6 (2013). Article ID 651495. https://doi.org/10.1155/2013/651495

6. Pavkovic, B. J., Kamenarovic, I.: The equiform differential geometry of curves in the Galilean space $G_{3}$. Glas. Mat. 22(42), 449-457 (1987)

7. Divjak, E. Z. B.: The equiform differential geometry of curves in the pseudo-Galilean space. Math. Commun. 13(2), 321-332 (2008)

8. Nawratil, G.: Quaternionic approach to equiform kinematics and line-elements of Euclidean 4-space and 3-space. Comput. Aided Geom. Design. 47, 150-162 (2016)

9. Pavkovic, B. J.: Equiform geometry of curves in the isotropic spaces $I_{1}^{3}$ and $I_{2}^{3}$. Rad JAZU, $39-44$ (1986)

10. El-sayied, H. K., Elzawy, M., Elsharkawy, A.: Equiform spacelike normal curves according to equiform-Bishop frame in $E_{1}^{3}$. Math. Meth. Appl. Sci. 17 (2017). https://doi.org/10.1002/mma.4618

11. El-sayied, H. K., Elzawy, M., Elsharkawy, A.: Equiform timelike normal curves in Minkowski space $E_{1}^{3}$. Far East. J. Math. Sci. 101, 1619-1629 (2017)

12. O'Neill, B.: Semi-Riemannian Geometry with Applications to Relativity. Academic Press, New York (1983)

13. Walrave, J.: Curves and surfaces in Minkowski space. Doctoral thesis. Leuven, K.U. Faculty of Science, Leuven (1995)

\section{Publisher's Note}

Springer Nature remains neutral with regard to jurisdictional claims in published maps and institutional affiliations.

\section{Submit your manuscript to a SpringerOpen ${ }^{\circ}$ journal and benefit from:}

- Convenient online submission

Rigorous peer review

- Open access: articles freely available online

High visibility within the field

- Retaining the copyright to your article

Submit your next manuscript at $\gg$ springeropen.com 